\title{
On sandwich theorems for some subclasses defined by generalized hypergeometric functions
}

Ekram Elsayed Ali, Rabha Mohamed El-Ashwah and Mohamed Kamal Aouf

\begin{abstract}
In this paper we derive some subordination and superordination results for certain normalized analytic functions in the open unit disc, which are acted upon by a class of generalized hypergeometric function $H_{q, s}\left(\alpha_{1}\right)$.
\end{abstract}

Mathematics Subject Classification (2010): 30C45, 30C80.

Keywords: Differential subordination, superordination, hypergeometric function.

\section{Introduction}

Let $H$ be the class of analytic functions in the open unit $\operatorname{disc} U=\{z \in \mathbb{C}:|z|<1\}$ and let $H[a, n]$ denotes the subclass of the functions $f \in H$ of the form

$$
f(z)=a+a_{n} z^{n}+a_{n+1} z^{n+1}+\ldots \quad(a \in \mathbb{C}),
$$

and we let

$$
A_{m}=\left\{f \in H, f(z)=z+a_{m+1} z^{m+1}+a_{m+2} z^{m+2}+\ldots\right\} .
$$

Also, let $A_{1}=A$ be the subclass of the functions $f \in H$ of the form

$$
f(z)=z+a_{2} z^{2}+\ldots
$$

For $f, g \in H$, we say that the function $f$ is subordinate to $g$, written symbolically as follows:

$$
f \prec g \quad \text { or } \quad f(z) \prec g(z),
$$

if there exists a Schwarz function $w$, which (by definition) is analytic in $\mathrm{U}$ with $w(0)=0$ and $|w(z)|<1,(z \in U)$, such that $f(z)=g(w(z))$ for all $z \in U$. In particular, if the function $g(z)$ is univalent in $U$, then we have the following equivalence (cf., e.g., [15]; see also [16, p.4]):

$$
f(z) \prec g(z) \Leftrightarrow f(0) \prec g(0) \text { and } \quad f(U) \subset g(U) .
$$


Supposing that $p$ and $h$ are two analytic functions in $U$, let

$$
\varphi(r, s, t ; z): \mathbb{C}^{3} \times U \rightarrow \mathbb{C} .
$$

If $p$ and $\varphi\left(p(z), z p^{\prime}(z), z^{2} p^{\prime \prime}(z) ; z\right)$ are univalent functions in $U$ and if $p$ satisfies the second-order superordination

$$
h(z) \prec \varphi\left(p(z), z p^{\prime}(z), z^{2} p^{\prime \prime}(z) ; z\right),
$$

then $p$ is called to be a solution of the differential superordination (1.3). (If $f$ is subordinate to $F$, then $F$ is superordination to $f$ ). An analytic function $q$ is called a subordinant of (1.3), if $q(z) \prec p(z)$ for all the functions $p$ satisfying (1.3). A univalent subordinant $\widetilde{q}$ that satisfies $q \prec \widetilde{q}$ for all of the subordinants $q$ of (1.3), is called the best subordinant (cf., e.g.,[15], see also [16]).

Recently, Miller and Mocanu [17] obtained sufficient conditions on the functions $h, q$ and $\varphi$ for which the following implication holds:

$$
k(z) \prec \varphi\left(p(z), z p^{\prime}(z), z^{2} p^{\prime \prime}(z) ; z\right) \Rightarrow q(z) \prec p(z) .
$$

Using the results Miller and Mocanu [17], Bulboaca [5] considered certain classes of first-order differential superordinations as well as superordination preserving integral operators [4]. Ali et al. [1], have used the results of Bulboaca [5] and obtained sufficient conditions for certain normalized analytic functions $f$ to satisfy

$$
q_{1}(z) \prec \frac{z f^{\prime}(z)}{f(z)} \prec q_{2}(z),
$$

where $q_{1}$ and $q_{2}$ are given univalent functions in $U$ with $q_{1}(0)=1$. Shanmugam et al. [23] obtained sufficient conditions for normalized analytic functions $f$ to satisfy

$$
q_{1}(z) \prec \frac{f(z)}{z f^{\prime}(z)} \prec q_{2}(z)
$$

and

$$
q_{1}(z) \prec \frac{z^{2} f^{\prime}(z)}{\{f(z)\}^{2}} \prec q_{2}(z),
$$

where $q_{1}$ and $q_{2}$ are given univalent functions in $U$ with $q_{1}(0)=1$ and $q_{2}(0)=1$, while Obradovic and Owa [20] obtained subordination results with the quantity $\left(\frac{f(z)}{z}\right)^{\mu}$. A detailed investigation of starlike functions of complex order and convex functions of complex order using Briot-Bouquet differential subordination technique has been studied very recently by Srivastava and Lashin [26] (see also [27], [2] and [19]).

For complex parameters

$$
\alpha_{1}, \ldots, \alpha_{q} \text { and } \beta_{1}, \ldots, \beta_{s} \quad\left(\beta_{j} \notin \mathbb{Z}_{0}^{-}=\{0,-1,-2, \ldots\} ; j=1,2, \ldots, s\right),
$$

we now define the generalized hypergeometric function ${ }_{q} F_{s}\left(\alpha_{1}, \ldots, \alpha_{q} ; \beta_{1}, \ldots, \beta_{s} ; z\right)$ by (see, for example, [25, p.19])

$$
\begin{aligned}
& { }_{q} F_{s}\left(\alpha_{1}, \ldots, \alpha_{q} ; \beta_{1}, \ldots, \beta_{s} ; z\right)=\sum_{k=0}^{\infty} \frac{\left(\alpha_{1}\right)_{k} \ldots\left(\alpha_{q}\right)_{k}}{\left(\beta_{1}\right)_{k} \ldots\left(\beta_{s}\right)_{k}} \cdot \frac{z^{k}}{k !} \\
& \left(q \leq s+1 ; q, s \in \mathbb{N}_{0}=\mathbb{N} \cup\{0\}, \mathbb{N}=\{1,2, \ldots\} ; z \in U\right),
\end{aligned}
$$


where $(\theta)_{\nu}$ is the Pochhammer symbol defined, in terms of the Gamma function $\Gamma$, by

$$
(\theta)_{\nu}=\frac{\Gamma(\theta+\nu)}{\Gamma(\theta)}= \begin{cases}1 & \left(\nu=0 ; \theta \in \mathbb{C}^{*}=\mathbb{C} \backslash\{0\}\right), \\ \theta(\theta-1) \ldots(\theta+\nu-1) & (\nu \in \mathbb{N} ; \theta \in \mathbb{C}) .\end{cases}
$$

Corresponding to the function $h\left(\alpha_{1}, \ldots, \alpha_{q} ; \beta_{1}, \ldots, \beta_{s} ; z\right)$, defined by

$$
h\left(\alpha_{1}, \ldots, \alpha_{q} ; \beta_{1}, \ldots, \beta_{s} ; z\right)=z_{q} F_{s}\left(\alpha_{1}, \ldots, \alpha_{q} ; \beta_{1}, \ldots, \beta_{s} ; z\right),
$$

Dziok and Srivastava [9] ( see also [10]) considered a linear operator

$$
H\left(\alpha_{1}, \ldots, \alpha_{q} ; \beta_{1}, \ldots, \beta_{s} ; z\right): A \rightarrow A,
$$

which is defined by the following Hadamard product (or convolution):

$$
H\left(\alpha_{1}, \ldots, \alpha_{q} ; \beta_{1}, \ldots, \beta_{s} ; z\right) f(z)=h\left(\alpha_{1}, \ldots, \alpha_{q} ; \beta_{1}, \ldots, \beta_{s} ; z\right) * f(z) .
$$

We observe that, for a function $f(z) \in A_{m}$, we have

$$
H\left(\alpha_{1}, \ldots, \alpha_{q} ; \beta_{1}, \ldots, \beta_{s}\right) f(z)=z+\sum_{k=m+1}^{\infty} \frac{\left(\alpha_{1}\right)_{k-1} \ldots\left(\alpha_{q}\right)_{k-1}}{\left(\beta_{1}\right)_{k-1} \ldots\left(\beta_{s}\right)_{k-1}(1)_{k-1}} a_{k} z^{k} .
$$

For $m=1$, we have (see [9])

$$
H\left(\alpha_{1}, \ldots, \alpha_{q} ; \beta_{1}, \ldots, \beta_{s}\right) f(z)=z+\sum_{k=2}^{\infty} \frac{\left(\alpha_{1}\right)_{k-1} \ldots\left(\alpha_{q}\right)_{k-1}}{\left(\beta_{1}\right)_{k-1} \ldots\left(\beta_{s}\right)_{k-1}(1)_{k-1}} a_{k} z^{k}
$$

For convenience, we write

$$
H_{q, s}\left(\alpha_{1}\right)=H\left(\alpha_{1}, \ldots, \alpha_{q} ; \beta_{1}, \ldots, \beta_{s}\right) .
$$

It is easily follows from (1.7) that (see [9])

$$
z\left(H_{q, s}\left(\alpha_{1}\right) f(z)\right)^{\prime}=\alpha_{1} H_{q, s}\left(\alpha_{1}+1\right) f(z)-\left(\alpha_{1}-1\right) H_{q, s}\left(\alpha_{1}\right) f(z) .
$$

It should be remarked that the linear operator $H_{q, s}\left(\alpha_{1}\right)$ is a generalization of many other linear operators considered earlier. In particular for $f \in A$ we have the following observation:

(i) $H_{2,1}(a, b ; c) f(z)=I_{c}^{a, b} f(z)\left(a, b \in \mathbb{C} ; c \notin \mathbb{Z}_{0}^{-}\right)$, where the linear operator $I_{c}^{a, b}$ was investigated by Hohlov [12];

(ii) $H_{2,1}(\delta+1,1 ; 1) f(z)=D^{\delta} f(z)(\delta>-1)$, where $D^{\delta}$ is the Ruscheweyh derivative of $f(z)$ (see $[22])$;

(iii) $H_{2,1}(\mu+1,1 ; \mu+2) f(z)=\mathcal{F}_{\mu}(f)(z)=\frac{\mu+1}{z^{\mu}} \int_{0}^{z} t^{\mu-1} f(t) d t(\mu>-1)$, where $\mathcal{F}_{\mu}$ is the Libera integral operator (see [13], [14] and [3]);

(iv) $H_{2,1}(a, 1 ; c) f(z)=L(a, c) f(z)\left(a \in \mathbb{R} ; c \in \mathbb{R} \backslash \mathbb{Z}_{0}^{-}\right)$, where $L(a, c)$ is the Carlson-Shaffer operator (see [6]);

(v) $H_{2,1}(\lambda+1, c ; a) f(z)=I^{\lambda}(a, c) f(z)\left(a, c \in \mathbb{R} \backslash \mathbb{Z}_{0}^{-} ; \lambda>-1\right)$, where $I^{\lambda}(a, c) f(z)$ is the Cho-Kwon-Srivastava operator (see [7]);

(vi) $H_{2,1}(\mu, 1 ; \lambda+1) f(z)=I_{\lambda, \mu} f(z)(\lambda>-1 ; \mu>0)$, where $I_{\lambda, \mu} f(z)$ is the Choi-Saigo-Srivastava operator [8] which is closely related to the Carlson-Shaffer [6] operator $L(\mu, \lambda+1) f(z)$;

(vii) $H_{2,1}(1,1 ; n+1) f(z)=I_{n} f(z)\left(n \in \mathbb{N}_{0}\right)$, where $I_{n} f(z)$ is the Noor operator of $n-t h$ order (see [18]); 
92 Ekram Elsayed Ali, Rabha Mohamed El-Ashwah and Mohamed Kamal Aouf

(viii) $H_{2,1}(2,1 ; 2-\mu) f(z)=\Omega_{z}^{\mu} f(z)(\mu \neq 2,3,4, \ldots)$, where $\Omega_{z}^{\mu}$ is the fractional derivative operator (see Owa and Srivastava [21]).

\section{Preliminaries}

In order to prove our subordination and superordination results, we make use of the following known definition and lemmas.

Definition 2.1. [17] Denote by $Q$ the set of all functions $f(z)$ that are analytic and injective on $\bar{U} \backslash E(f)$, where

$$
E(f)=\left\{\zeta: \zeta \in \partial U \text { and } \lim _{z \rightarrow \zeta} f(z)=\infty\right\},
$$

and are such that $f^{\prime}(\zeta) \neq 0$ for $\zeta \in \partial U \backslash E(f)$.

Lemma 2.2. [16] Let the function $q(z)$ be univalent in the unit disc $U$, and let $\theta$ and $\varphi$ be analytic in a domain $D$ containing $q(U)$, with $\varphi(w) \neq 0$ when $w \in q(U)$. Set $Q(z)=z q^{\prime}(z) \varphi(q(z)), h(z)=\theta(q(z))+Q(z)$ and suppose that

(i) $Q$ is a starlike function in $U$,

(ii) $\operatorname{Re}\left(\frac{z h^{\prime}(z)}{Q(z)}\right)>0$ for $z \in U$.

If $p$ is analytic in $U$ with $p(0)=q(0), p(U) \subseteq D$ and

$$
\theta(p(z))+z p^{\prime}(z) \varphi(p(z)) \prec \theta(q(z))+z q^{\prime}(z) \varphi(q(z)),
$$

then $p(z) \prec q(z)$, and $q$ is the best dominant.

Lemma 2.3. [16] Let $g$ be a convex function in $U$ and let

$$
h(z)=g(z)+m \alpha z g^{\prime}(z),
$$

where $\alpha>0$ and $m$ is a positive integer. If

$$
p(z)=g(0)+p_{m} z^{m}+\ldots .
$$

is analytic in $U$ and

$$
p(z)+\alpha z p^{\prime}(z) \prec h(z),
$$

then

and this result is sharp.

$$
p(z) \prec g(z),
$$

Lemma 2.4. [11] Let $h$ be a convex function with $h(0)=a$ and let $\gamma \in \mathbb{C}$ with $\operatorname{Re}(\gamma) \geq$ 0. If $p \in H$ with $p(0)=a$ and

$$
p(z)+\frac{1}{\gamma} z p^{\prime}(z) \prec h(z)
$$

then

$$
p(z) \prec q(z) \prec h(z),
$$

where

$$
q(z)=\frac{\gamma}{n z^{(\gamma / n)}} \int_{0}^{z} h(t) t^{(\gamma / n)-1} d t \quad(z \in U) .
$$

The function $q$ is convex and is the best dominant. 
Lemma 2.5. [4] Let $q(z)$ be a convex univalent function in the unit disc $U$ and let $\vartheta$ and $\varphi$ be analytic in a domain D containing $q(U)$. Suppose that

(i) $\operatorname{Re}\left\{\frac{\vartheta^{\prime}(q(z))}{\varphi(q(z))}\right\}>0$ for $z \in U$;

(ii) $z q^{\prime}(z) \varphi(q(z))$ is starlike in $U$.

If $p \in H[q(0), 1] \cap Q$ with $p(U) \subseteq D$, and $\vartheta(p(z))+z p^{\prime}(z) \varphi(p(z))$ is univalent in $U$, and

$$
\vartheta(q(z))+z q^{\prime}(z) \varphi(q(z)) \prec \vartheta(p(z))+z p^{\prime}(z) \varphi(p(z)),
$$

then $q(z) \prec p(z)$, and $q$ is the best subordinant.

\section{Subordination results for analytic functions}

Unless otherwise mentioned we shall assume throughout the paper that $q \leq$ $s+1 ; q, s \in \mathbb{N}_{0}, \mu, \beta \in \mathbb{C}^{*}, \eta, \alpha, \delta, \xi \in \mathbb{C}, z \in U$ and the powers understood as principle values.

Theorem 3.1. Let the function $q$ be analytic and univalent in $U$, with $q(z) \neq 0(z \in$ $\left.U^{*}=U \backslash\{0\}\right)$. Suppose that $\frac{z q^{\prime}(z)}{q(z)}$ is starlike univalent in $U$. Let

$$
\operatorname{Re}\left\{1+\frac{\xi}{\beta} q(z)+\frac{2 \delta}{\beta}(q(z))^{2}-\frac{z q^{\prime}(z)}{q(z)}+\frac{z q^{\prime \prime}(z)}{q^{\prime}(z)}\right\}>0 \quad(z \in U) .
$$

If $f \in A_{m}$ and $q$ satisfies the following subordination:

$$
\Psi\left(f, \alpha_{1}, \alpha, \delta, \xi, \beta, \mu, \eta\right) \prec \alpha+\xi q(z)+\delta(q(z))^{2}+\beta \frac{z q^{\prime}(z)}{q(z)},
$$

where

$$
\begin{gathered}
\Psi\left(f, \alpha_{1}, \alpha, \delta, \xi, \beta, \mu\right)=\alpha+\xi\left(\frac{H_{q, s}\left(\alpha_{1}\right) f(z)}{z}\right)^{\mu}\left(\frac{z}{H_{q, s}\left(\alpha_{1}+1\right) f(z)}\right)^{\eta} \\
+\delta\left(\frac{H_{q, s}\left(\alpha_{1}\right) f(z)}{z}\right)^{2 \mu}\left(\frac{z}{H_{q, s}\left(\alpha_{1}+1\right) f(z)}\right)^{2 \eta}+\beta \mu \alpha_{1}\left[\frac{H_{q, s}\left(\alpha_{1}+1\right) f(z)}{H_{q, s}\left(\alpha_{1}\right) f(z)}-1\right] \\
+\beta \eta\left(\alpha_{1}+1\right)\left[1-\frac{H_{q, s}\left(\alpha_{1}+2\right) f(z)}{H_{q, s}\left(\alpha_{1}+1\right) f(z)}\right]
\end{gathered}
$$

then

$$
\left(\frac{H_{q, s}\left(\alpha_{1}\right) f(z)}{z}\right)^{\mu}\left(\frac{z}{H_{q, s}\left(\alpha_{1}+1\right) f(z)}\right)^{\eta} \prec q(z)
$$

and $q$ is the best dominant of (3.2).

Proof. Define the function $p$ by

$$
p(z)=\left(\frac{H_{q, s}\left(\alpha_{1}\right) f(z)}{z}\right)^{\mu}\left(\frac{z}{H_{q, s}\left(\alpha_{1}+1\right) f(z)}\right)^{\eta} \quad(z \in U) .
$$

Then the function $p(z)$ is analytic in $U$ and $p(0)=1$. Differentiating (3.4) logarithmically with respect to $z$, we have

$$
\frac{z p^{\prime}(z)}{p(z)}=\mu\left[\frac{z\left(H_{q, s}\left(\alpha_{1}\right) f(z)\right)^{\prime}}{H_{q, s}\left(\alpha_{1}\right) f(z)}-1\right]+\eta\left[1-\frac{z\left(H_{q, s}\left(\alpha_{1}+1\right) f(z)\right)^{\prime}}{H_{q, s}\left(\alpha_{1}+1\right) f(z)}\right] .
$$


94 Ekram Elsayed Ali, Rabha Mohamed El-Ashwah and Mohamed Kamal Aouf

By using the identity (1.8) in the resulting equation, we have

$$
\frac{z p^{\prime}(z)}{p(z)}=\mu\left[\frac{\alpha_{1} H_{q, s}\left(\alpha_{1}+1\right) f(z)}{H_{q, s}\left(\alpha_{1}\right) f(z)}-\alpha_{1}\right]+\eta\left[\left(\alpha_{1}+1\right)-\frac{\left(\alpha_{1}+1\right) H_{q, s}\left(\alpha_{1}+2\right) f(z)}{H_{q, s}\left(\alpha_{1}+1\right) f(z)}\right] \text {. }
$$

By setting

$$
\theta(w)=\alpha+\xi w(z)+\delta w^{2}(z) \quad \text { and } \quad \phi(w)=\frac{\beta}{w},
$$

it can be easily observed that $\theta$ is analytic in $\mathbb{C}, \phi$ is analytic in $\mathbb{C}^{*}$ and that $\phi(w) \neq$ $0\left(w \in \mathbb{C}^{*}\right)$. Also, by letting

$$
Q(z)=z q^{\prime}(z) \phi(q(z))=\beta \frac{z q^{\prime}(z)}{q(z)}
$$

and

$$
h(z)=\theta\{q(z)\}+Q(z)=\alpha+\xi q(z)+\delta(q(z))^{2}+\beta \frac{z q^{\prime}(z)}{q(z)},
$$

we find that $Q$ is starlike univalent in $U$ and that

$$
\operatorname{Re}\left(\frac{z h^{\prime}(z)}{Q(z)}\right)=\operatorname{Re}\left\{1+\frac{\xi}{\beta} q(z)+\frac{2 \delta}{\beta}(q(z))^{2}-\frac{z q^{\prime}(z)}{q(z)}+\frac{z q^{\prime \prime}(z)}{q^{\prime}(z)}\right\}>0 .
$$

The assertion (3.5) of Theorem 3.1 now follows by an application of Lemma 2.2.

Putting $q(z)=\frac{1+A z}{1+B z},(-1 \leq B<A \leq 1)$ in Theorem 3.1, we obtain the following corollary.

\section{Corollary 3.2. Suppose that}

$\operatorname{Re}\left\{1+\frac{\xi}{\beta}\left(\frac{1+A z}{1+B z}\right)+\frac{2 \delta}{\beta}\left(\frac{1+A z}{1+B z}\right)^{2}-\frac{(A-B) z}{(1+A z)(1+B z)}-\frac{2 B z}{1+B z}\right\}>0(z \in U)$.

If $f \in A_{m}$ satisfies the subordination

$$
\Psi\left(f, \alpha_{1}, \alpha, \delta, \xi, \beta, \mu, \eta\right) \prec \alpha+\xi\left(\frac{1+A z}{1+B z}\right)+\delta\left(\frac{1+A z}{1+B z}\right)^{2}+\frac{\beta(A-B) z}{(1+A z)(1+B z)},
$$

where $\Psi\left(f, \alpha_{1}, \alpha, \delta, \xi, \beta, \mu, \eta\right)$ is given by (3.3), then

$$
\left(\frac{H_{q, s}\left(\alpha_{1}\right) f(z)}{z}\right)^{\mu}\left(\frac{z}{H_{q, s}\left(\alpha_{1}+1\right) f(z)}\right)^{\eta} \prec \frac{1+A z}{1+B z}
$$

and $\frac{1+A z}{1+B z}$ is the best dominant.

corollary.

Putting $q(z)=\left(\frac{1+z}{1-z}\right)^{\gamma}(0<\gamma \leq 1)$ in Theorem 3.1, we obtain the following

Corollary 3.3. Suppose that

$$
\operatorname{Re}\left\{1+\frac{\xi}{\beta}\left(\frac{1+z}{1-z}\right)^{\gamma}+\frac{2 \delta}{\beta}\left(\frac{1+z}{1-z}\right)^{2 \gamma}-\frac{2 \gamma z}{1-z^{2}}+\frac{2 z(\gamma+z)}{(1-z)(1+z)}\right\}>0 \quad(z \in U) .
$$

If $f \in A_{m}$ satisfies the subordination

$$
\Psi\left(f, \alpha_{1}, \alpha, \delta, \xi, \beta, \mu, \eta\right) \prec \alpha+\xi\left(\frac{1+z}{1-z}\right)^{\gamma}+\delta\left(\frac{1+z}{1-z}\right)^{2 \gamma}+\frac{2 \gamma \beta z}{1-z^{2}},
$$


where $\Psi\left(f, \alpha_{1}, \alpha, \delta, \xi, \beta, \mu, \eta\right)$ is given by (3.3), then

$$
\left(\frac{H_{q, s}\left(\alpha_{1}\right) f(z)}{z}\right)^{\mu}\left(\frac{z}{H_{q, s}\left(\alpha_{1}+1\right) f(z)}\right)^{\eta} \prec\left(\frac{1+z}{1-z}\right)^{\gamma}
$$

and $\left(\frac{1+z}{1-z}\right)^{\gamma}$ is the best dominant.

Putting $q(z)=e^{\mu A z}$, with $|\mu A|<\pi$ in Theorem 3.1, we obtain the following corollary.

\section{Corollary 3.4. Suppose that}

$$
\operatorname{Re}\left\{1+\frac{\xi}{\beta} e^{\mu A z}+\frac{2 \delta}{\beta} e^{2 \mu A z}\right\}>0 \quad(z \in U) .
$$

If $f(z) \in A_{m}$ satisfies the subordination

$$
\Psi\left(f, \alpha_{1}, \alpha, \delta, \xi, \beta, \mu\right) \prec \alpha+\xi e^{\mu A z}+\delta e^{2 \mu A z}+\beta \mu A z,
$$

where $\Psi\left(f, \alpha_{1}, \alpha, \delta, \xi, \beta, \mu, \eta\right)$ is given by (3.3), then

$$
\left(\frac{H_{q, s}\left(\alpha_{1}\right) f(z)}{z}\right)^{\mu}\left(\frac{z}{H_{q, s}\left(\alpha_{1}+1\right) f(z)}\right)^{\eta} \prec e^{\mu A z}
$$

and $e^{\mu A z}$ is the best dominant.

Remark 3.5. (i) Putting $q=2, s=1, \alpha_{1}=\alpha_{2}=\beta_{1}=1, m=1, \delta=\xi=\eta=0$, $\beta=\frac{1}{\mu}$ and $q(z)=e^{\mu A z}$, with $|\mu A|<\pi$ in Theorem 3.1, we obtain the result obtained by Obradovic and Owa [20];

(ii) Putting $q(z)=\frac{1}{(1-z)^{2 b}}\left(b \in \mathbb{C}^{*}\right), q=2, s=1, \alpha_{1}=\alpha_{2}=\beta_{1}=1, m=1$, $\delta=\xi=\eta=0, \mu=\alpha=1$ and $\beta=\frac{1}{b}$ in Theorem 3.1, we obtain the result obtained by Srivastava and Lashin [26];

(iii) Putting $q(z)=\frac{1}{(1-z)^{2 a b}}\left(a, b \in \mathbb{C}^{*}\right), q=2, s=1, \alpha_{1}=\alpha_{2}=\beta_{1}=1, m=1$, $\delta=\xi=\eta=0, \mu=\alpha=1$ and $\beta=\frac{1}{a b}$ in Theorem 3.1, we obtain the result obtained by Obradovic et al.[19];

(iv) Putting $q(z)=(1+B z)^{\mu(A-B) / B},\left(\mu \in \mathbb{C}^{*},-1 \leq B<A \leq 1\right), q=2, s=1$, $\alpha_{1}=\alpha_{2}=\beta_{1}=1, m=1, \beta=\frac{1}{\mu}, \alpha=1$ and $\delta=\xi=\eta=0$ in Theorem 3.1, we obtain the result obtained by Obradovic and Owa [20];

(v) Putting $q(z)=(1-z)^{-2 a b \cos \lambda e^{-i \lambda}}\left(a, b \in C^{*},|\lambda|<\frac{\pi}{2}\right), \beta=\frac{e^{i \lambda}}{a b \cos \lambda}, q=2$, $s=1, \alpha_{1}=\alpha_{2}=\beta_{1}=1, m=1, \delta=\xi=\eta=0$ and $\mu=\alpha=1$ in Theorem 3.1, we obtain the result obtained by Aouf et al. [2, Theorem 1].

Theorem 3.6. Let $h \in H, h(0)=1, h^{\prime}(0) \neq 0$ which satisfy

$$
\operatorname{Re}\left\{1+\frac{z h^{\prime \prime}}{(z)} h^{\prime}(z)\right\}>-\frac{1}{2} \quad(z \in U) \text {. }
$$

If $f(z) \in A_{m}$ satisfies the differential subordination:

$$
\frac{H_{q, s}\left(\alpha_{1}+k\right) f(z)}{z} \prec h(z) \quad\left(k \in \mathbb{Z}^{+} ; z \in U^{*}\right),
$$

then

$$
\frac{H_{q, s}\left(\alpha_{1}+k-1\right) f(z)}{z} \prec g(z) \quad\left(k \in \mathbb{Z}^{+}\right)
$$


96 Ekram Elsayed Ali, Rabha Mohamed El-Ashwah and Mohamed Kamal Aouf

where

$$
g(z)=\frac{\left(\alpha_{1}+k-1\right)}{m z^{\left(\alpha_{1}+k-1\right) / m}} \int_{0}^{z} h(t) t^{\left(\left(\alpha_{1}+k-1\right) / m\right)-1} d t \quad\left(k \in \mathbb{Z}^{+}\right) .
$$

The function $g$ is convex and is the best dominant.

Proof. Let the function $p(z)$ be defined by

$$
p(z)=\frac{H_{q, s}\left(\alpha_{1}+k-1\right) f(z)}{z} \quad\left(k \in \mathbb{Z}^{+}\right) .
$$

Then the function $p$ is analytic in $U$ and $p(0)=1$. Differentiating (3.8) logarithmically with respect to $z$, we have

$$
\frac{z p^{\prime}(z)}{p(z)}=\left[\frac{z\left(H_{q, s}\left(\alpha_{1}+k-1\right) f(z)\right)^{\prime}}{H_{q, s}\left(\alpha_{1}+k-1\right) f(z)}-1\right] \quad\left(k \in \mathbb{Z}^{+}\right) .
$$

By using the identity (1.8), we have

$$
\frac{z p^{\prime}(z)}{p(z)}=\left[\frac{\left(\alpha_{1}+k-1\right) H_{q, s}\left(\alpha_{1}+k\right) f(z)}{H_{q, s}\left(\alpha_{1}+k-1\right) f(z)}-\left(\alpha_{1}+k-1\right)\right]
$$

and hence

$$
p(z)+\frac{z p^{\prime}(z)}{\alpha_{1}+k-1}=\frac{H_{q, s}\left(\alpha_{1}+k\right) f(z)}{z} \quad\left(k \in \mathbb{Z}^{+}\right) .
$$

The assertion of Theorem 3.6 now follows by applying Lemma 2.4.

Putting $k=1$ in Theorem 3.6, we get.

Corollary 3.7. If $f \in A_{m}$ satisfies the differential subordination:

$$
\frac{H_{q, s}\left(\alpha_{1}+1\right) f(z)}{z} \prec h(z)
$$

then

where

$$
\frac{H_{q, s}\left(\alpha_{1}\right) f(z)}{z} \prec g(z)
$$

$$
g(z)=\frac{\alpha_{1}}{m z^{\alpha_{1} / m}} \int_{0}^{z} h(t) t^{\left(\alpha_{1} / m\right)-1} d t \quad(z \in U) .
$$

The function $g(z)$ is convex and is the best dominant.

By using Lemma 2.3 we can prove the following theorem.

Theorem 3.8. Let $g$ be convex function with $g(0)=1$. Let $h$ be a function, such that

$$
h(z) \prec g(z)+\frac{m}{\lambda+1} z g^{\prime}(z) .
$$

If $f \in A_{m}$ satisfies the subordination:

$$
\frac{H_{q, s}\left(\alpha_{1}+k\right) f(z)}{z} \prec h(z) \quad\left(k \in \mathbb{Z}^{+}\right),
$$

then

and is the best dominant.

$$
\frac{H_{q, s}\left(\alpha_{1}+k-1\right) f(z)}{z} \prec g(z) \quad\left(k \in \mathbb{Z}^{+}\right),
$$


Proof. The proof of Theorem 3.8 is much akin to the proof of Theorem 3.6 and hence we omit the details involved.

Next, by appealing to Lemma 2.5, we prove Theorem 3.9.

Theorem 3.9. Let $q$ be analytic and convex univalent in $U$, such that $q(z) \neq 0$ and $\frac{z q^{\prime}(z)}{q(z)}$ be starlike univalent in $U$. Further, let us assume that

$$
\operatorname{Re}\left\{\frac{2 \delta}{\beta}(q(z))^{2}+\frac{\xi}{\beta} q(z)\right\}>0 \quad(z \in U) .
$$

If $f \in A_{m}, 0 \neq\left(\frac{H_{q, s}\left(\alpha_{1}\right) f(z)}{z}\right)^{\mu}\left(\frac{z}{H_{q, s}\left(\alpha_{1}+1\right) f(z)}\right)^{\eta} \in H[q(0), 1], \Psi\left(f, \alpha_{1}, \alpha, \delta, \xi, \beta, \mu, \eta\right)$ is univalent in $U$, where $\Psi\left(f, \alpha_{1}, \alpha, \delta, \xi, \beta, \mu\right)$ is given by (3.3), and

$$
\alpha+\xi q(z)+\delta(q(z))^{2}+\beta \frac{z q^{\prime}(z)}{q(z)} \prec \Psi\left(f, \alpha_{1}, \alpha, \delta, \xi, \beta, \mu, \eta\right),
$$

then

$$
q(z) \prec\left(\frac{H_{q, s}\left(\alpha_{1}\right) f(z)}{z}\right)^{\mu}\left(\frac{z}{H_{q, s}\left(\alpha_{1}+1\right) f(z)}\right)^{\eta}
$$

and $q$ is the best subordinant of (3.11).

Proof. By setting

$$
\vartheta(z)=\alpha+\xi w+\delta w^{2} \quad \text { and } \quad \varphi(w)=\beta \frac{w^{\prime}}{w},
$$

it is easily observed that $\vartheta$ is analytic in $\mathbb{C}$. Also, $\varphi$ is analytic in $\mathbb{C}^{*}$ and that $\varphi(w) \neq$ $0\left(w \in \mathbb{C}^{*}\right)$.

Since $q$ is convex (univalent) function it follows that,

$$
\operatorname{Re}\left\{\frac{\vartheta^{\prime}(q(z))}{\varphi(q(z))}\right\}=\operatorname{Re}\left\{\frac{2 \delta}{\beta}(q(z))^{2}+\frac{\xi}{\beta} q(z)\right\}>0 \quad(z \in U) .
$$

The assertion (3.12) of Theorem 3.9 follows by an application of Lemma 2.5.

\section{Sandwich result}

Combining Theorem 3.1 and Theorem 3.9, we get the following sandwich theorem.

Theorem 4.1. Let $q_{1}$ be convex univalent and $q_{2}$ be univalent in $U$ such that $q_{1}(z) \neq 0$ and $q_{2}(z) \neq 0$. Suppose $q_{1}$ satisfies (3.10) and $q_{2}$ satisfies (3.1). If $f \in A_{m}$, $0 \neq\left(\frac{H_{q, s}\left(\alpha_{1}\right) f(z)}{z}\right)^{\mu}\left(\frac{z}{H_{q, s}\left(\alpha_{1}+1\right) f(z)}\right)^{\eta} \in H[q(0), 1]$, and $\Psi\left(f, \alpha_{1}, \alpha, \delta, \xi, \beta, \mu, \eta\right)$ is univalent in $U$ and satisfies

$$
\begin{aligned}
\alpha+\xi q_{1}(z)+\delta\left(q_{1}(z)\right)^{2}+\beta \frac{z q_{1}^{\prime}(z)}{q_{1}(z)} & \prec \Psi\left(f, \alpha_{1}, \alpha, \delta, \xi, \beta, \mu, \eta\right) \\
& \prec \alpha+\xi q_{2}(z)+\delta\left(q_{2}(z)\right)^{2}+\beta \frac{z q_{2}^{\prime}(z)}{q_{2}(z)},
\end{aligned}
$$

where $\Psi\left(f, \alpha_{1}, \alpha, \delta, \xi, \beta, \mu, \eta\right)$ is given by (3.3), then

$$
q_{1}(z) \prec\left(\frac{H_{q, s}\left(\alpha_{1}\right) f(z)}{z}\right)^{\mu}\left(\frac{z}{H_{q, s}\left(\alpha_{1}+1\right) f(z)}\right)^{\eta} \prec q_{2}(z)
$$


and $q_{1}$ and $q_{2}$ are, respectively, the best subordinant and best dominant.

Remark 4.2. (i) Putting $q=2, s=1, \alpha_{1}=a(a>0), \alpha_{2}=1$ and $\beta_{1}=c(c>0)$ in our results we will improve all results obtained by Shanmugam et al. [24];

(ii) By specializing the parameters $q, s, \alpha_{i}\left(\alpha_{1}, \ldots, \alpha_{q}\right)$ and $\beta_{j}\left(\beta_{1}, \ldots, \beta_{s}\right)$ in our results, we obtain the corresponding results due to various operators mentioned in the introduction.

Acknowledgments. The authors thanks the referee for their valuable suggestions which led to improvement of this study.

\section{References}

[1] Ali, R.M., Ravichandran, V., Khan, M.H., Subramaniam, K.G., Differential sandwich theorems for certain analytic functions, Far East J. Math. Sci., 15(2004), no. 1, 87-94.

[2] Aouf, M.K., Al-Oboudi, F.M., Haidan, M.M., On some results for $\lambda$-spirallike and $\lambda$ Robertson functions of complex order, Publ. Institute Math. Belgrade, 77(2005), no. 91, 93-98.

[3] Bernardi, S.D., Convex and starlike univalent functions, Trans. Amer. Math. Soc., 135(1969), 429-446.

[4] Bulboacă, T., A class of superordination preserving integral operators, Indag. Math. (New Series), 13(2002), no. 3, 301-311.

[5] Bulboacă, T., Classes of first-order differential subordinations, Demonstratio Math., 35(2002), no. 2, 287-392.

[6] Carlson, B.C., Shaffer, D.B., Starlike and prestarlike hypergeometric functions, SIAM J. Math. Anal., 15(1984), 737-745.

[7] Cho, N.E., Kwon, O.H., Srivastava, H.M., Inclusion and argument properties for certain subclasses of multivalent functions associated with a family of linear operators, J. Math. Anal. Appl., 292(2004), 470-483.

[8] Choi, J.H., Saigo, M., Srivastava, H.M., Some inclusion properties of a certain family of integral operators, J. Math. Anal. Appl., 276(2002), 432-445.

[9] Dziok, J., Srivastava, H.M., Classes of analytic functions associated with the generalized hypergeometric function, Appl. Math. Comput., 103(1999), 1-13.

[10] Dziok, J., Srivastava, H.M., Certain subclasses of analytic functions associated with the generalized hypergeometric function, Integral Transform. Spec. Funct., 14(2003), 7-18.

[11] Hallenbeck, D.J., Ruscheweyh, St., Subordination by convex functions, Proc. Amer. Math. Soc., 52(1975), 191-195.

[12] Hohlov, Yu.E., Operators and operations in the class of univalent functions, (in Russian), Izv. Vysšh. Učebn. Zaved. Mat., 10(1978), 83-89.

[13] Libera, R.J., Some classes of regular univalent functions, Proc. Amer. Math. Soc., 16(1965), 755-658.

[14] Livingston, A.E., On the radius of univalence of certain analytic functions, Proc. Amer. Math. Soc., 17(1966), 352-357.

[15] Miller, S.S., Mocanu, P.T., Differential subordinations and univalent functions, Michigan Math. J., 28(1981), 157-171. 
[16] Miller, S.S., Mocanu, P.T., Differential Subordinations Theory and Applications, Series on Monographs and Textbooks in Pure and Applied Math., No. 225, Marcel Dekker Inc. New York, Basel, 2000.

[17] Miller, S.S., Mocanu, P.T., Subordinations of differential superordinations, Complex Variables, 48(2003), no. 10, 815-826.

[18] Noor, K.I., On new classes of integral operator, J. Nat. Geom., 16(1999), no. 1-2, 71-80.

[19] Obradovic, M., Aouf, M.K., Owa, S., On some results for starlike functions of complex order, Publ. Inst. Math. Belgrade, 46(1989), no. 60, 79-85.

[20] Obradovic, M., Owa, S., On certain properties for some classes of starlike functions, J. Math. Anal. Appl., 145(1990), no. 2, 357-364.

[21] Owa, S., Srivastava, H.M., Univalent and starlike generalized hypergeometric functions, Canad. J. Math., 39(1987), 1057-1077.

[22] Ruscheweyh, St., New criteria for univalent functions, Proc. Amer. Math. Soc., 49(1975), 109-115.

[23] Shanmugam, T.N., Ravichandran, V., Sivasubramanian, S., Differential sandwich theorems for some subclasses of analyitc functions, Austral. J. Math. Anal. Appl., 3(2006), no. 1, 1-11.

[24] Shanmugam, T.N., Sivasubramanian, S., Owa, S., On sandwich results for some subclasses of analytic functions involving certain linear operator, Integral Transform. Spec. Funct., 21(2010), no. 1, 1-11.

[25] Srivastava, H.M., Karlsson, P.W., Multiple Gaussian Hypergeometric Series, Ellis Horwood Ltd., Chichester, Halsted Press (John Wiley \& Sons, Inc.), New York, 1985.

[26] Srivastava, H.M., Lashin, A.Y., Some applications of the Briot-Bouquet differential subordination, J. Inequal. Pure Appl. Math., 6(2005), no. 2, 1-7.

[27] Tuneski, N., On certain sufficient conditions for starlikeness, Internat. J. Math. Math. Sci., 23(2000), no. 8, 521-527.

Ekram Elsayed Ali

Department of Mathematics and Computer Science

Faculty of Science, Port-Said University

Port Said, 42521, Egypt

e-mail: ekram_008eg@yahoo.com

Rabha Mohamed El-Ashwah

Department of Mathematics

Faculty of Science, Damietta University

New Damiette, 34517, Egypt

e-mail: r_elashwah@yahoo.com

Mohamed Kamal Aouf

Department of Mathematics

Faculty of Science, Mansoura University

Mansoura 35516, Egypt

e-mail: mkaouf 127@yahoo.com 